\title{
Ipilimumab Activity in Advanced Uveal Melanoma: A Pooled Analysis
}

\author{
Muhammad A Khattak ${ }^{1,2,3^{*}}$ and Elin Gray Ka $^{3,4}$ \\ ${ }^{1}$ Fiona Stanley Hospital, Perth, Western Australia, Australia \\ ${ }^{2}$ University of Western Australia, Perth, Western Australia, Australia \\ ${ }^{3}$ Edith Cowan University, Perth, Western Australia, Australia \\ ${ }^{4}$ Lions Eye Institute, Perth, Western Australia, Australia
}

* Corresponding author: Dr. Muhammad Adnan Khattak, Department of Medical Oncology/University of Western Australia, Fiona Stanley Hospital, 11 Robin Warren Drive, Murdoch, WA, Australia, Tel: +61410565868, E-mail: adnan.khattak@ health.wa.gov.au

\begin{abstract}
Background: Uveal Melanoma is a rare tumour that displays different clinical behavior and molecular features compared with cutaneous melanoma. It is generally resistant to systemic therapy and there is no current standard effective therapy to treat patients with advanced disease.

Patients and methods: We searched Medline, PubMed, EMBASE and major oncology conference abstracts from the past 5 years to identify relevant studies evaluating ipilimumab monotherapy in uveal melanoma. Data were extracted on ipilimumab dose, sample size, Objective Response Rate (ORR), Progression Free Survival (PFS), median Overall Survival (mOS), Disease Control Rate (DCR), 1 year Overall Survival (1yrOS) and 2 year Overall Survival (2yrOS).

Results: Nine studies were included in this study including Phase II clinical trials $(n=2)$, Expanded Access Programs $($ EAP) $(n=4)$ and retrospective studies $(n=3)$. Cases were a mix of pre-treated and treatment-naïve patients. Reported mOS ranged from 5.2-28 months (median: 9.3 months), and reported $1 \mathrm{yrOS}$ ranged between $4.5-65 \%$. Calculated compound ORR was $3.4 \%$, and compound DCR was $36 \%$.

Conclusion: Ipilimumab has limited clinical activity in advanced uveal melanoma. Further research is needed to identify more effective systemic therapies for management of these patients.
\end{abstract}

\section{Keywords}

Ipilimumab, Uveal melanoma, Anti-CTLA4

\section{Introduction}

Uveal Melanoma (UM) is a rare tumour, with an estimated annual global incidence between 5.3-10.9 cases per million people [1]. It accounts for less than $5 \%$ of all melanoma cases [2]. UM arises from the vascular layers of the eye (iris, ciliary body, and choroid). It is a highly aggressive malignancy and about $50 \%$ of patients will develop metastatic disease at some stage after prior diagnosis of primary uveal melanoma [3]. Liver is the dominant site of metastases (60-90\%) followed by lungs $(25 \%)[4,5]$. Prognosis in advanced disease is poor, with median overall survival ranging between 2-15 months [5-7].

UM has a distinct molecular profile, and displays different clinical behavior to cutaneous melanoma. In contrast to BRAF and NRAS mutations commonly seen in cutaneous melanoma, mutations in heterotrimeric $\mathrm{G}$ protein- $\alpha$ (GNA) subunits GNAQ and GNA11 have been reported in up to $80 \%$ of uveal melanomas [8].

Treatment options for UM are particularly limited and efficacy data is scarce [9]. There are a few small studies that describe options such as chemotherapy, metastasectomy, and hepatic intra-arterial chemoembolization [10]. Response rates are dismal [9], with one retrospective review reporting response rates to systemic treatment being as low as $1 \%$ [3].

Immunotherapy has revolutionized the treatment landscape for metastatic melanoma in the last 5 years. Ipilimumab is a fully humanized monoclonal antibody that blocks the Cytotoxic T-Lymphocyte-Associated Antigen 4 (CTLA-4), promoting anti-tumour immunity. It is routinely used in metastatic melanoma, and was one

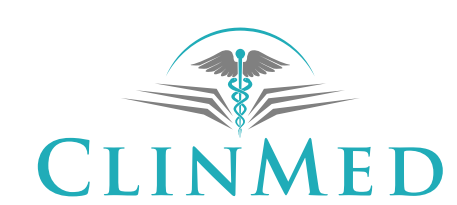

INTERNATIONAL LIBRARY ir

Citation: Khattak MA, Gray E (2017) Ipilimumab Activity in Advanced Uveal Melanoma: A Pooled Analysis. Int J Cancer Clin Res 4:088. doi.org/10.23937/2378-3419/1410088

Received: September 03, 2017; Accepted: November 16, 2017; Published: November 18, 2017

Copyright: (C) 2017 Khattak MA, et al. This is an open-access article distributed under the terms of the Creative Commons Attribution License, which permits unrestricted use, distribution, and reproduction in any medium, provided the original author and source are credited. 
of the first treatment to ever demonstrate a survival benefit in large randomized trial in advanced melanoma $[11,12]$. However, most of the studies examining activity of ipilimumab in advanced melanoma have traditionally excluded patients with uveal melanoma $[11,12]$.

The aim of this paper was to conduct pool analysis of published literature, evaluating the efficacy of ipilimumab monotherapy in the management of advanced uveal melanoma.

\section{Patients and Methods}

This review was conducted in accordance with the Preferred Reporting Items for Systematic Reviews and Meta-Analyses (PRISMA) guidelines [13].

MEDLINE, PubMed and EMBASE search was conducted to identify relevant articles, with no restrictions placed on the search. All published prospective and retrospective studies, examining ipilimumimab activity in advanced uveal melanoma, amongst both pre-treated and treatment-naïve patients were included. Relevant abstracts from the American Society of Clinical Oncology (ASCO) and European Society of Medical Oncology (ESMO) annual scientific meetings presented in the last 5 years were also evaluated. We used the following search terms: "Uveal melanoma" and "ipilimumab", in any combination in the titles of published trials or conference abstracts. Initial search results were screened for duplication and relevance. The full articles and abstracts were then reviewed in detail. Data were manually extracted on study design, ipilimumab dose, sample size, Objective Response Rate (ORR), Progression Free Survival (PFS), Overall Survival (OS), Disease Control Rate (DCR), 1 year Overall Survival (1yrOS) and 2 year Overall Survival (2yrOS). Note was made of any limitations and bias present in individual studies.

Our primary endpoints were median Overall Survival (mOS) and percentage 1 year Overall Survival (1yrOS). Secondary endpoints included Objective Response Rate (ORR), Disease Control Rate (DCR) and median Progression Free Survival (mPFS).

Due to wide variation in study design and reported outcomes, key efficacy endpoints from each individual trial were summarized. Forest plots were constructed to depict and compare PFS and OS, along with 95\% Confidence Intervals $(95 \% \mathrm{Cl})$ for each trial where relevant data was available. Pooled data was used to calculate compound ORR and DCR using a random distribution model.

\section{Results}

The identification and screening process as per PRIM-

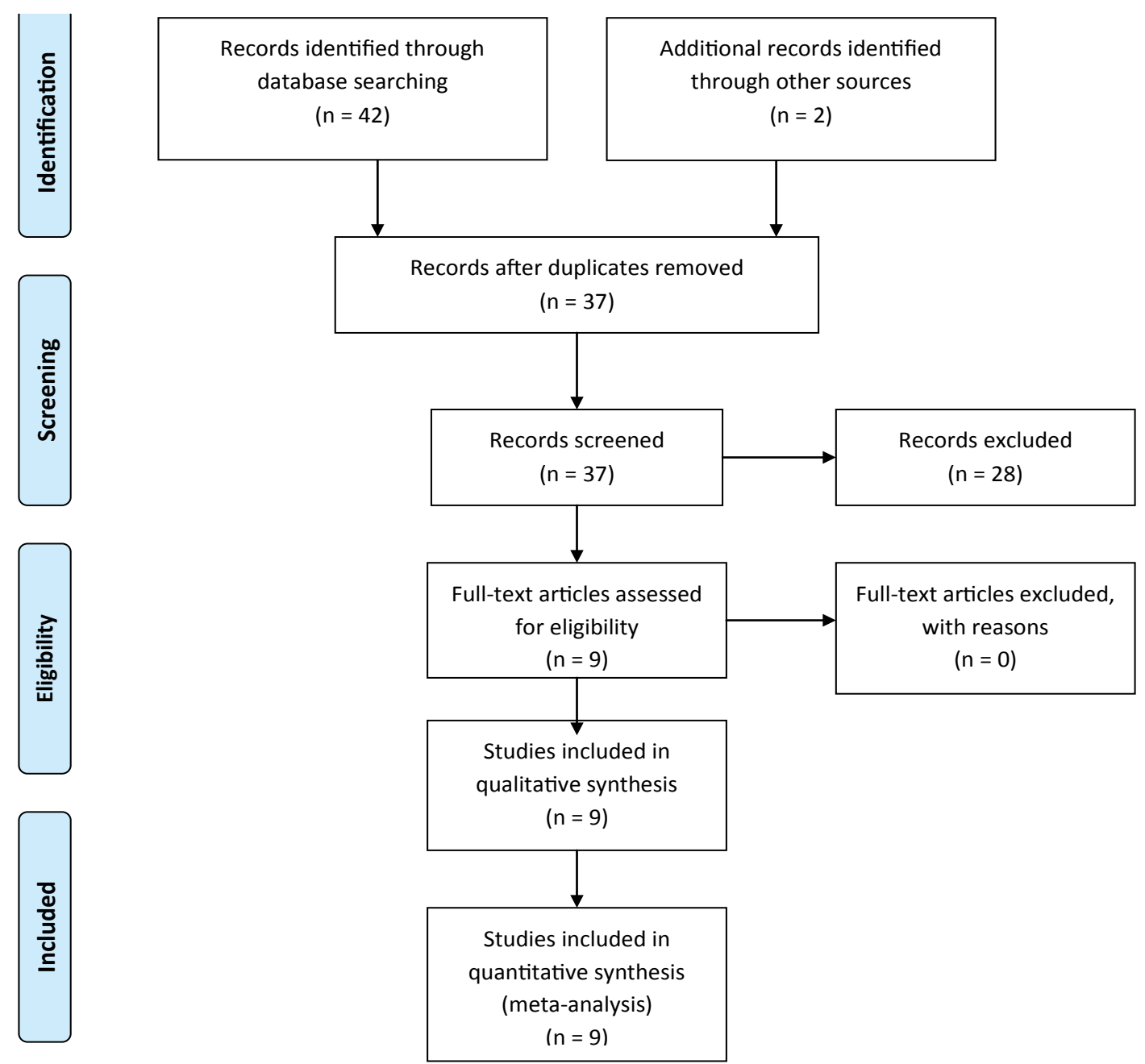

Figure 1: Study Identification, Screening and Inclusion process (PRISMA flow chart). 
SA guidelines is depicted in Figure 1. Nine articles were included in this study; including Phase II clinical trials ( $\mathrm{n}$ $=2)$, Expanded Access Programs (EAP) $(n=4)$ and retrospective studies $(n=3)$ [14-22]. The key features of each trial are summarized in Table 1.

The reported median patient age ranged from 42-67 years. Ipilimumab was prescribed at either a high induction dose $(10 \mathrm{mg} / \mathrm{kg}$ ) or standard dose $(3 \mathrm{mg} / \mathrm{kg})$. Reported mOS ranged from 5.2-28 months, with an overall median of 9.3 months (mean of 9.1 months) (Figure 2). Progression free survival data was limited and ranged from 2.83.6 (Figure 3). Median PFS could not be calculated for the reported studies as relevant data was not published or not provided. The 1yr OS ranged between 4.5-65\% (Figure 4). Calculated compound ORR was $3.4 \%$ and compound DCR was $36 \%$.

Eight studies included a toxicity analysis, with immune related adverse effects (irAEs) graded according to common toxicity criteria. Common irAEs included rash, itch, diarrhea/colitis, hepatitis, and hypophysitis,

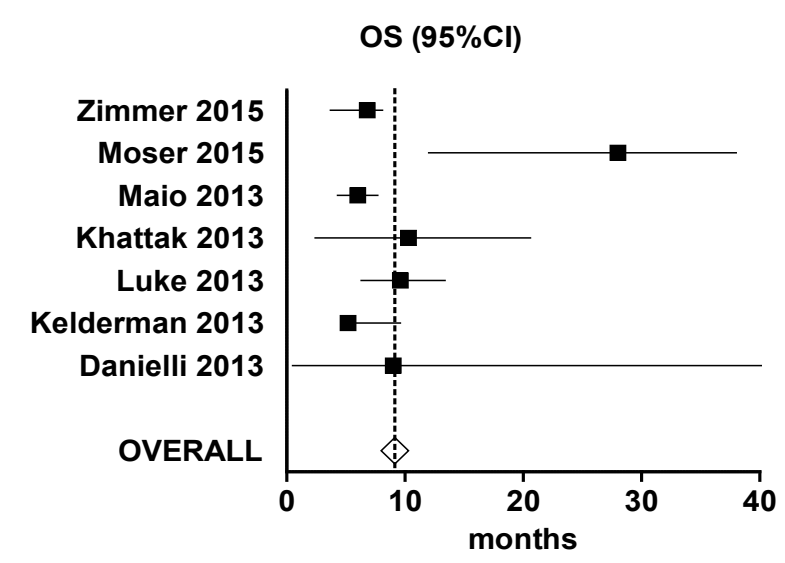

Figure 2: Forest plot of median Overall Survival (OS) from included trials. in addition to fatigue and fever. Grade 3 or 4 irAEs occurred in $10-33 \%$ of cases. IrAE's were generally reversible when managed as per protocol-specific guidelines. No new safety signals were seen compared to original ipilimumab phase 3 studies.

\section{Discussion}

Uveal Melanoma is a rare and highly aggressive tu-

PFS $(95 \% \mathrm{Cl})$

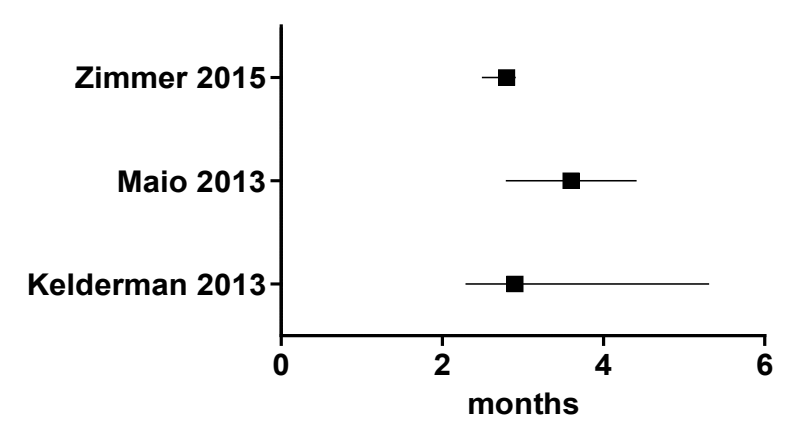

Figure 3: Forest plot of progression free survival from included trials.

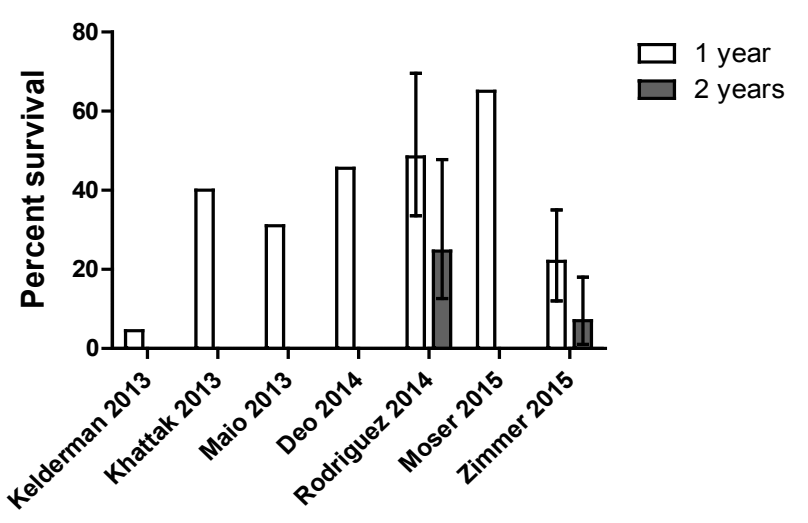

Figure 4: Survival \% at 1 and 2 years for reported trials.

Table 1: Summary of trials included in meta-analysis.

\begin{tabular}{|c|c|c|c|c|c|c|c|c|c|c|}
\hline Study & Design & $\mathbf{N}$ & $\begin{array}{l}\text { Ipi dose } \\
\text { (mg/kg) }\end{array}$ & Pre-treated & ORR\% & DCR\% & mPFS & mOS & 1yr OS\% & 2yr OS\% \\
\hline Zimmer [15] & Phase II & 53 & 3 & Mixed & 0 & 47 & $\begin{array}{l}2.8 \\
(2.5-2.9)\end{array}$ & $\begin{array}{l}6.8 \\
(3.7-8.1)\end{array}$ & $\begin{array}{l}22 \\
(12-35)\end{array}$ & $\begin{array}{l}7 \\
(1-18)\end{array}$ \\
\hline Rodriguez [16] & Phase II & 32 & 10 & Rx Naive & $\begin{array}{l}6.4 \\
(0.79-21.4)\end{array}$ & 45.1 & NR & NR & $\begin{array}{l}48.4 \\
(33.6-69.6)\end{array}$ & $\begin{array}{l}24.6 \\
(12.6-47.8)\end{array}$ \\
\hline Deo [14] & Retro & 24 & 3 & Pre-Rx & 4 & 21 & 2.8 & 9.7 & 45.6 & 11.4 \\
\hline Maio [17] & EAP & 82 & 3 & Pre-Rx & 5 & 34 & $\begin{array}{l}3.6 \\
(2.8-4.4)\end{array}$ & $\begin{array}{l}6 \\
(4.3-7.7)\end{array}$ & 31 & NR \\
\hline Khattak [18] & EAP & 5 & 3 & Pre-Rx & 0 & 40 & NR & $\begin{array}{l}10.3 \\
(2.4-20.6)\end{array}$ & 40 & NR \\
\hline Luke [19] & Retro & 39 & $3 / 10$ & Mixed & 2.6 & 46.1 & NR & $\begin{array}{l}9.6 \\
(6.3-13.4)\end{array}$ & NR & NR \\
\hline Kelderman [20] & EAP & 22 & 3 & Pre-Rx & 4.5 & 9 & $\begin{array}{l}2.9 \\
(2.3-5.3)\end{array}$ & $\begin{array}{l}5.2 \\
(4.9-9.6)\end{array}$ & 4.5 & NR \\
\hline Danielli [21] & EAP & 13 & 10 & Pre-Rx & 0 & 23 & NR & $\begin{array}{l}9 \\
(0.5-43)\end{array}$ & NR & NR \\
\hline Moser [22] & NR & 23 & NR & Pre-Rx & NR & NR & NR & $\begin{array}{l}28 m \\
(12-38)\end{array}$ & 65 & NR \\
\hline
\end{tabular}

NR: Not Reported; ORR: Objective Response Rate; PFS: Progression Free Survival; OS: Overall Survival; DCR: Disease Control Rate. 
mour, with limited treatment options. Data on the activity of ipilimumab amongst patients with advanced UM is scarce. This pooled analysis contributes to the existing literature by summarizing the key efficacy endpoints in published trials and abstracts.

In terms of our activity endpoints, the reported efficacy of ipilimumab in UM is modest at best. However, median overall survival of 9.3 months amongst patients with UM treated with ipilimumab (range 5.2-28 months) is similar to the published mOS of cutaneous melanoma patients treated with ipilimumab [11,12]. The calculated compound ORR was disappointingly low (3.4\%), but the compound DCR was potentially more promising (36\%).

Most of the published data assessing the efficacy of ipilimumab focuses on response rate and median overall survival as primary endpoints. At least one abstract suggests that survival at 1-2 years is more relevant to assess the efficacy of ipilimumab, given the highly aggressive nature of the disease [14]. The long term benefit with ipilimumab in metastatic melanoma is noted by a consistent absolute increase of approximately $10 \%$ of patients alive in the ipilimumab-containing arms compared to the control therapy in the two pivotal clinical trials $[11,12]$. The late plateau or tail represents the long term responders. Therefore survival at certain time points like 1 and 2 yr OS\% are more meaningful in the context of advanced UM. The 1 year OS\% with ipilimumab in advanced UM in our analysis ranged between $4.5-65 \%$.

Importantly, the toxicity profile described by these studies was comparable to the description and frequency of irAEs among patients with cutaneous melanoma treated with equivalent doses of ipilimumab. Whilst serious adverse events (grade $3 / 4$ ) were common, they were easily managed and usually reversible when treated as per study protocol with steroids.

More recently, there have been studies with anti-programmed cell death protein 1 antibody (anti-PD1) monotherapy and targeted therapy with MEK inhibitors that have demonstrated some activity [23-25]. Although at least one small series has observed activity with pembrolizumab, more extensive experience suggests that responses and clinical benefit are much more limited than with advanced cutaneous melanoma $[23,24]$. The role of the newer agents in the treatment landscape for advanced UM needs to be determined and clinically justified bearing in mind the toxicity seen especially with targeted therapy [25].

One major limitation to this analysis is the heterogeneity of the studies selected. There was a lack of consistency between studies and the wide variation in study design, inclusion criteria and response assessment criteria is reflected in the wide variation in reported outcomes. There was limited consistency in choice of primary and secondary efficacy outcomes, with confidence intervals not reported in several studies. We were unable to retrieve more complete data by contacting the primary investigators despite our efforts to do so. Sample size in all these studies is relatively small and most are retrospective. It is possible that the inclusion of results from EAPs predisposes investigators to a positive publication bias.

\section{Conclusion}

Despite the limitations of the published data, we think there is reasonable evidence to suggest that ipilimumab has modest clinical activity and manageable toxicity when used in the treatment of advanced UM in the absence of any other suitable systemic therapy options. Future studies should explore newer immunotherapies and targeted therapies either as monotherapy or in combination.

\section{References}

1. Papastefanou VP, Cohen VML (2011) Uveal melanoma. J Skin Cancer 2011: 573974.

2. Singh AD, Turell ME, Topham AK (2011) Uveal melanoma: Trends in incidence, treatment, and survival. Ophthalmology 118: 1881-1885.

3. Bedikian AY, Legha SS, Mavligit G, Carrasco CH, Khorana S, et al. (1995) Treatment of uveal melanoma metastatic to the liver: A review of the M. D. Anderson Cancer Center experience and prognostic factors. Cancer 76: 1665-1670.

4. Kujala E, Mäkitie T, Kivelä T (2003) Very long-term prognosis of patients with malignant uveal melanoma. Invest Ophthalmol Vis Sci 44: 4651-4659.

5. Gragoudas ES, Egan KM, Seddon JM, Glynn RJ, Walsh SM, et al. (1991) Survival of patients with metastases from uveal melanoma. Ophthalmology 98: 383-389.

6. Rietschel P, Panageas KS, Hanlon C, Patel A, Abramson $\mathrm{DH}$, et al. (2005) Variates of survival in metastatic uveal melanoma. J Clin Oncol 23: 8076-8080.

7. Augsburger JJ, Corrêa ZM, Shaikh AH (2009) Effectiveness of treatments for metastatic uveal melanoma. Am J Ophthalmol 148: 119-127.

8. Van Raamsdonk CD, Bezrookove V, Green G, Bauer J, Gaugler L, et al. (2009) Frequent somatic mutations of GNAQ in uveal melanoma and blue naevi. Nature 457: 599-602.

9. Pereira PR, Odashiro AN, Lim LA, Miyamoto C, Blanco PL, et al. (2013) Current and emerging treatment options for uveal melanoma. Clin Ophthalmol 7: 1669-1682.

10. Woodman SE (2012) Metastatic uveal melanoma: biology and emerging treatments. Cancer J 18: 148-152.

11. Hodi FS, O'Day SJ, McDermott DF, Weber RW, Sosman JA, et al. (2010) Improved survival with ipilimumab in patients with metastatic melanoma. N Engl J Med 363: 711-723.

12. Robert C, Thomas L, Bondarenko I, O'Day S, Weber J, et al. (2011) Ipilimumab plus dacarbazine for previously untreated metastatic melanoma. N Engl J Med 364: 25172526.

13. Moher D, Liberati A, Tetzlaff J, Altman DG, PRISMA Group (2009) Preferred reporting items for systematic reviews and meta-analyses: the PRISMA statement. Ann Intern Med 151: 264-269. 
14. Deo MA (2014) Long-term survival benefit from ipilimumab treatment in metastatic uveal melanoma patients. In ASCO Annual Meeting Proceedings 32: 3060.

15. Zimmer L, Vaubel J, Mohr P, Hauschild A, Utikal J, et al (2015) Phase II DeCOG-Study of Ipilimumab in Pretreated and Treatment-Naïve Patients with Metastatic Uveal Melanoma. PLoS One 10: e0118564.

16. Piulats Rodriguez JM, Ochoa de Olza M, Codes M, Lopez-Martin JA, Berrocal A, et al. (2014) Phase II study evaluating ipilimumab as a single agent in the first-line treatment of adult patients (Pts) with metastatic uveal melanoma (MUM): The GEM-1 trial. ASCO Meet Abstr 32: 9033.

17. Maio M, Danielli R, Chiarion-Sileni V, Pigozzo J, Parmiani G, et al. (2013) Efficacy and safety of ipilimumab in patients with pre-treated, uveal melanoma. Ann Oncol 24: 29112915.

18. Khattak MA, Fisher R, Hughes P, Gore M, Larkin J (2013) Ipilimumab activity in advanced uveal melanoma. Melanoma Res 23: 79-81.

19. Luke JJ, Callahan MK, Postow MA, Romano E, Ramaiya $\mathrm{N}$, et al. (2013) Clinical activity of ipilimumab for metastatic uveal melanoma: A retrospective review of the Dana-Farber Cancer Institute, Massachusetts General Hospital, Memorial Sloan-Kettering Cancer Center, and University Hospital of Lausanne experience. Cancer 119: 3687-3695.
20. Kelderman S, van der Kooij MK, van den Eertwegh AJ, Soetekouw PM, Jansen RL, et al. (2013) Ipilimumab in pretreated metastastic uveal melanoma patients. Results of the Dutch Working group on Immunotherapy of Oncology (WIN-O). Acta Oncol 52: 1786-1788.

21. Danielli R, Ridolfi R, Chiarion-Sileni $V$, Queirolo $P$, Testori A, et al. (2012) Ipilimumab in pretreated patients with metastatic uveal melanoma: Safety and clinical efficacy. Cancer Immunol Immunother 61: 41-48.

22. Moser JC, Pulido JS, Dronca RS, McWilliams RR, Markovic SN, et al. (2015) The Mayo Clinic experience with the use of kinase inhibitors, ipilimumab, bevacizumab, and local therapies in the treatment of metastatic uveal melanoma. Melanoma Res 25: 59-63.

23. Kottschade LA, McWilliams RR, Markovic SN, Block MS, Villasboas Bisneto J, et al. (2016) The use of pembrolizum$\mathrm{ab}$ for the treatment of metastatic uveal melanoma. Melanoma Res 26: 300-303.

24. Algazi AP, Tsai KK, Shoushtari AN, Munhoz RR, Eroglu Z, et al. (2016) Clinical outcomes in metastatic uveal melanoma treated with PD-1 and PD-L1 antibodies. Cancer 122: 3344-3353.

25. Carvajal RD, Sosman JA, Quevedo JF, Milhem MM, Joshua $A M$, et al. (2014) Effect of selumetinib vs chemotherapy on progression-free survival in uveal melanoma: A randomized clinical trial. JAMA 311: 2397-2405. 